\title{
A multivariate modeling for analysis of factors controlling the particle size and viscosity in palm kernel oil esters-based nanoemulsions
}

\begin{abstract}
An artificial neural network (ANN) was used to develop predictive models for studying and identifying factors that influence particle size and viscosity of sodium diclofenac-loaded palm kernel oil esters-nanomeulsions. The effect of four independent variables namely water content, oil and surfactant $(\mathrm{O} / \mathrm{S})$ ratio, mixing rate and mixing time were considered as inputs of the network trained. The particle size and viscosity of samples in various compositions prepared under different rate and time of high shear emulsification, were measured as output. Data, split into training, testing and validating sets, were modeled by incremental back propagation (IBP) algorithm. The developed model represents high quality performance of the neural network and its capability in modeling and identifying the critical factors that control preparation of the nanoemulsions. Water content with $30.82 \%$ importance was found to be the main parameter controlling the particle size and viscosity in the system, followed by $\mathrm{O} / \mathrm{S}$ ratio, mixing rate and mixing time, with 27.28, 22.06 and $19.84 \%$ importance, respectively. The model was then employed to investigate the effect of composition and processing factors on particle size and viscosity of the nanoemulsions.
\end{abstract}

Keyword: Artificial neural network; Nanoemulsions; Palm kernel oil esters; Drug delivery; Particle size; Viscosity 